\title{
Construyendo la modernidad \\ a través del deporte espectáculo. El Gran Premio de México, 1962-1970
}

\author{
Building modernity through sport as a show.
}

The Mexican Grand Prix, 1962-1970

\section{Hugo Alejandro Avendaño Santoyo*}

\section{Licenciado y maestro en Sociolo- gía por la UNAM, México. Docto- rante en Historia por la Universi- dad Iberoamericana Ciudad de México. Profesor de asignatura en la Facultad de Ciencias Políticas y Sociales de la UNAM. Miembro del Seminario de Historia de la Edu- cación Física y los Deportes en México, del Instituto Mora. -alejandro.avsan@gmail.com http://orcid.org/0000-0002- 9507-5536}

\section{RECIBID O: 25.7.2021}

\section{Resumen}

El proceso de modernización en México tuvo una naturaleza multidimensional que no se limitó a la estructura productiva del país. El tiempo de ocio fue un importante bastión ideológico del proyecto modernizador impulsado por las élites políticas y económicas surgidas luego de la Revolución mexicana. Los medios de comunicación, las artes y el deporte espectáculo se convirtieron, durante la segunda mitad del siglo XX, en importantes escenarios ideológicos en los que se construyeron las narrativas e identidades que acompañaron la formación de la nación mexicana moderna. Se analiza el Gran Premio de México de Fórmula 1 (1962-1970), un espectáculo deportivo que motivó la profesionalización del automovilismo deportivo mexicano. Este acontecimiento expuso la integración de las élites políticas y económicas como dirigentes de un proyecto país que agotaba el modelo keynesiano. A su vez, formó parte de una serie de eventos deportivos que construyeron una narrativa y una identidad gráfica que apoyaron la construcción de la nación mexicana. Así, se propone una interpretación sociohistórica de este evento que lo piensa como parte de dinámicas políticas y económicas interrelacionadas, en la que se vislumbran personajes clave que encarnan valores y contradicciones propios del momento histórico. 
Palabras clave: deporte, modernización, sociología del tiempo libre, México.

\section{Abstract}

The modernization process in Mexico had a multidimensional nature, not limited to the productive structure of the country. Leisure time was an important ideological bastion of the modernizing project promoted by the political and economic elites that emerged after the Mexican Revolution. The media, the arts and the sport as ashow became, during the second half of the twentieth century, important ideological scenarios where the narratives and identities that accompanied the formation of the modern Mexican nation were built. The article analyzes the Mexican Grand Prix of Formula 1 (1962-1970), a sporting spectacle that motivated the professionalization of Mexican motorsport. This event exposed the integration of political and economic elites as leaders of a country project that exhausted the Keynesian model. In turn, it was part of a series of sporting events that built a narrative and graphic identity which supported the construction of the Mexican nation. Thus, a socio-historical interpretation of this event is proposed, as part of a series of interrelated political and economic dynamics, in which key characters who embody values and contradictions typical of the historical moment are glimpsed. Keywords: sport, modernization, sociology of leisure time, Mexico.

\section{Las claves de la carrera}

El presente texto es producto de una investigación de corte sociohistórico cuyo interés se centra en un largo proceso de profesionalización identificado en el automovilismo deportivo practicado en México durante las décadas de los años cincuenta y sesenta del siglo XX. Durante este periodo, la práctica automovilística se profesionalizó no solo a través de la preparación física de los pilotos y el uso de máquinas más adecuadas para la competencia; también jugó a favor la organización de más eventos automovilísticos en el país, que empezaban a ser más populares luego de la celebración de la Carrera Panamericana durante los años cincuenta. ${ }^{1}$ Así, la actividad deportiva se considera como una estructura que mantiene una posición de interdependencia respecto a otros elementos constitutivos de la sociedad mexicana, como la economía, la política y la cultura. Se trata de explorar el grado y las formas de interrelación.

Para lograrlo, se recurre a una perspectiva figuracional, que se construye como un enfoque teórico basado en la identificación de redes de interdependencias entre estruc- 
turas y actores individuales y colectivos, a la luz de evidencia empírica recolectada alrededor de casos históricos que ilustran la transformación de las sociedades humanas (Ramos Torre, 1994, pp. 38-43). El análisis se nutre de fuentes periodísticas que cubrieron el Gran Premio de México entre 1962 y 1970: El Heraldo de México, El Universal, ESTO, Excélsior y Novedades. En conjunto con crónicas periodísticas elaboradas posteriormente por otros autores, se pudieron identificar actores individuales y colectivos clave para entender el proceso histórico de este evento, así como otros elementos estructurales que se relacionaron con él. Por último, el trabajo con estas fuentes permitió también la recolección de narrativas que ayudan a ilustrar estos procesos.

\section{La economía en México durante los años sesenta}

Desde una perspectiva macroeconómica, la década de los años sesenta del siglo XX observa la consolidación del desarrollo estabilizador (Gracida, 2004, p. 50). Ello fue posible por la política económica basada en la «expansión y estabilidad financiada por ahorro voluntario, crecientemente de origen externo» (Gracida, 2004, p. 50), así como un creciente proteccionismo que acercó entre sí a las élites políticas y económicas. La confianza de la clase empresarial se ganó no solo a través de la estabilidad cambiaria y de precios, sino también por la (forzada) estabilidad política derivada de la represión de movimientos sociales (Gracida, 2004, p. 50).

La estructura económica erigida en el México de la década de los años sesenta se convirtió en parte de un discurso político e ideológico que fue utilizado tanto hacia dentro como hacia el exterior del país. Hacia el exterior, el crecimiento económico (no estable ni igualitario) se consideró como un ejemplo de éxito y vanguardia. El sistema productivo mexicano se basó en la continuación de la sustitución progresiva del sector agrícola por el industrial, una tendencia que puede rastrearse desde los años cuarenta (de la Peña y Aguirre, 2004, pp. 392-394). Este modelo de producción fue posibilitado a su vez por la apertura de la economía a la inversión de capitales, la existencia de múltiples beneficios fiscales y también mediante la ampliación de la infraestructura nacional en los sectores energético, de telecomunicaciones y transportes (de la Peña y Aguirre, 2004, pp. 437-438). Asimismo, la economía nacional atrajo al capital extranjero a través de la industria turística y la venta de servicios en general (Cárdenas, 2012, p. 249).

Hacia el interior se generó un discurso triunfalista que acompañó estas transformaciones económicas. Dicho discurso apeló a «las capacidades de los mexicanos». Con esta herramienta ideológica, «se edificaron algunos de los más importantes relatos omnicomprensivos del siglo XX sobre la sociedad, el Estado y el individuo [mexicanos]» (Pozas Horcasitas, 2014, p. 224). Al mismo tiempo, durante este periodo «los gobiernos 
estimularon la desmovilización política y el conformismo, que se consideraban condiciones necesarias del crecimiento económico» (Pedraza Velásquez, 2011), mientras consolidaban también la estabilidad política como un valor central de la cultura democrática mexicana, en un círculo que terminaba por otorgarle legitimidad al régimen (Pozas Horcasitas, 2014, p. 52).

El Estado revolucionario, defensor de los valores institucionalizados de la Revolución mexicana (Pozas Horcasitas, 2014, pp. 49-50), generó una expresión narrativa de carácter nacionalista alrededor del «milagro mexicano». En ella también se manifestó una «cultura de la modernidad», necesaria para el bienestar de la nación (Loaeza, 1993, p. 113). Con todo lo anterior se estructuró un orden social fundado en ideales libertarios y nacionalistas, articulados por la acción colectiva al servicio de la clase dominante, la cual hizo pasar sus intereses de clase como el interés colectivo (Tello, 1979, pp. 203-205).

\section{La sociedad automotriz, génesis de la modernidad}

Entiendo el término sociedad automotriz como una descripción sociohistórica aplicable a la mayor parte de las sociedades modernas, cuya principal característica se encuentra en la masificación del automóvil, tanto en número como en formas de uso, así como en su introducción en las actividades sociales, culturales, políticas y económicas (Avendaño, 2019, pp. 39-41).

En su estructura se desarrolló la industria automotriz, la cual se fue convirtiendo paulatinamente en un sector importante para la estrategia de desarrollo económico nacional. El desarrollo de la industria estimuló el nacimiento de otros sectores industriales, algunos vinculados con los automóviles (Garrido Asperó, 2015, p. 110; Tovalín Ahumada, 2005, p. 23), además de transformar las cadenas de producción de otras actividades económicas. Eso fue posible gracias a la complicidad del sector con el Estado mexicano y sus planes de industrialización nacional. De esta manera, los capitales extranjeros pudieron establecer fábricas dentro del territorio nacional y lograr que el mercado interno captara en su totalidad la producción de estas, a cambio de entregar a los capitales nacionales el sector de autopartes y refacciones (Plana, 2004, pp. 110-112).

El automóvil también desencadenó la transformación espacial de los centros urbanos, que se reconfiguraron para privilegiar el nuevo flujo mercantil que aquel posibilitó. De esta manera, el automóvil se convirtió en un elemento cada vez más común para la vida social. Por otra parte, la invasión del automóvil orilló al peatón a desarrollar nuevas formas (marginales) de habitar la ciudad.

El automóvil también salió de la ciudad. Su potencial para el transporte de mercancías fue un argumento para colocarlo como un complemento de la red ferroviaria 
nacional (Jáuregui, 2004, p. 127), especialmente en un territorio complejo, con numerosas poblaciones prácticamente aisladas (Jáuregui, 2004, p. 127; Plana, 2004, p. 108). Así, «ya para 1912 se experimentaban los beneficios de transportar mercancías, se utilizaban automóviles para la persecución de maleantes en la ciudad de México y la Cruz Roja ya tenía ambulancias» (Jáuregui, 2004, p. 126).

Finalmente, en la sociedad automotriz el automóvil también adquiere un papel en el tiempo social de ocio. Las carreras de autos comenzaron prácticamente a la par del desarrollo de la tecnología automotriz. En México, las competencias automovilísticas adquirieron popularidad rápidamente $\mathrm{y}$, aunque ralentizadas por la Revolución mexicana, crecieron con la popularización del automóvil entre las clases medias, las que lograron institucionalizar también el llamado deporte motor.

De esta manera, el automóvil adquirió un papel protagónico en la escena ideológica y técnica del desarrollo económico, que a su vez moldeó al siglo XX en México y en el mundo. La máquina se transformó en uno de los más poderosos fetiches de la modernidad. La sociedad, ahora automotriz, transformó el significado de su tenencia, la cual se distingue «más como indicador social que como mercancía destinada a satisfacer el transporte individual [...] [y lo convierte en] el inequívoco signo del éxito en la cotidiana búsqueda del ascenso en la pirámide social» (Juárez Núñez et al., 2005, p. 11).

\section{El Gran Premio de México,}

\section{¿un precursor del deporte espectáculo moderno?}

La Fórmula 1 nació en Europa en 1950. Regulada por la Federación Internacional del Automóvil (FIA), se concibió como la competencia automovilística más importante del mundo. Las carreras que componen su campeonato retomaron su nombre (Gran Premio) de las competiciones automovilísticas previas a la Segunda Guerra Mundial. Dado que las armadoras automotrices más importantes del mundo estaban involucradas en esta competencia, la categoría fue adquiriendo importancia política y económica, particularmente a partir de los años sesenta.

La celebración del Gran Premio de México, como otros proyectos ideológicos, económicos y políticos de la época, fue posible gracias al interés de la clase empresarial, particularmente la mexicana. Esta tarea pudo haber sido especialmente facilitada por la cercanía con la clase gobernante, la cual estimaba a los empresarios como factores cada vez más importantes para la gobernabilidad y les abría las puertas para el emprendimiento de negocios con su cobijo (Jalife Villalón, 2015, pp. 234-235).

Entre los más cercanos al presidente Adolfo López Mateos se encontraba don Pedro Rodríguez Quijada, un hombre que había ascendido a través de negocios con Pemex 
y otras dependencias estatales (Jalife Villalón, 2015, pp. 36-37). Rodríguez era un entusiasta del automovilismo deportivo, al igual que el presidente, pero también era el padre y principal patrocinador de Pedro y Ricardo Rodríguez de la Vega, jóvenes pilotos que habían destacado en la comunidad automovilística. Mediante su amistad, convenció al entonces presidente de construir un autódromo donde se pudieran celebrar competencias internacionales, y que también sirviera como lugar de entrenamiento para sus hijos (Jalife Villalón, 2015, p. 117; Rosas, 2015, pp. 22-23).

La pista fue proyectada sobre la Ciudad Deportiva de la Magdalena Mixhuca, que había sido inaugurada en 1958 por Adolfo Ruiz Cortines. El trazado se basó en el proyecto que Óscar Fernández Gómez-Daza elaboró en 1955 para graduarse como ingeniero civil en la UNAM. ${ }^{2}$ La pista se inauguró el 20 de diciembre de 1959 con la celebración de una carrera de 500 kilómetros de duración ${ }^{3}$ (Hernández Padilla, 2015, p. 23), un espectáculo a escala nacional que logró convocar a «100 mil espectadores, 18 camarógrafos, mil elementos de tropa más 800 policías» (Rosas, 2015, p. 29).

A pesar de la existencia del nuevo autódromo, el país no contaba con suficientes competencias y pilotos que pudieran mantener la actividad automovilística durante todo el año en el recinto. Por otra parte, el corporativismo y la construcción de la Ciudad Deportiva promovían la práctica masiva del deporte en un espacio donde múltiples canchas $^{4}$ veían acción al mismo tiempo, lo que dificultaba la operación de la pista, que requería, por seguridad, que la actividad se pausara.

Los hermanos Pedro y Ricardo Rodríguez se encontraban corriendo desde 1957 en el extranjero (Jalife Villalón, 2015, pp. 66-74) y se habían convertido en importantes figuras de la esfera deportiva nacional e internacional. La entrada de Ricardo a Fórmula 1 en 1961 motivó a un grupo de inversionistas a buscar la celebración de una carrera de este campeonato. Entre ellos se encontraban Javier Velázquez, ${ }^{5}$ Carlos Braniff, ${ }^{6}$ Rómulo

2 Al respecto, recomiendo revisar Fernández Gómez-Daza (1955).

3 Tomando en cuenta que un Gran Premio de Fórmula 1 tiene una duración de 300 kilómetros, la prueba inaugural estuvo pensada para poner a prueba los automóviles, cuyas piezas solían tener una vida útil limitada. Por otra parte, el circuito medía 5 kilómetros, lo que se traducía en una competencia de 100 vueltas.

4 En 1959, la Ciudad Deportiva contaba con 23 canchas de básquetbol, un estadio de béisbol, 25 canchas de béisbol al aire libre adicionales, 7 canchas de fútbol, 43 canchas de fútbol adicionales y acondicionadas para ligas menores y 2 canchas para pelota mixteca. Contaba también con instalaciones para natación, clavados, tenis, tiro con arco, vóleibol, hockey sobre pasto y softbol (Rosas, 2015, p. 22).

5 Obtuvo victorias en pistas nacionales durante los años cincuenta. Fue el primer mexicano en competir en Sebring en 1955. Gracias a sus contactos en la política, se encargó, a través de su empresa de ingeniería, de construir el Autódromo de la Magdalena Mixhuca (Scudería Hermanos Rodríguez, 2007, p. 1).

6 También fue piloto de automovilismo. Formó el primer equipo mexicano que compitió en una prueba fuera de México, concretamente en las 12 Horas de Sebring de 1955 (Scudería Hermanos Rodríguez, 2007, p. 1). 
O’Farrill y Juan Manuel Rullán. Este grupo logró el aval de Adolfo López Mateos para celebrar en 1962 el I Gran Premio de México.?

El apoyo político del presidente López Mateos y su gobierno a los organizadores del Gran Premio de México fue público y bastante amplio (Hernández Padilla, 2015, p. 21; Jalife Villalón, 2015, pp. 234-235). La carrera, de exhibición, permitiría a la FIA evaluar las capacidades logísticas de los organizadores mexicanos con vistas a incluir la prueba en el calendario del campeonato a partir de 1963 (Rosas, 2015, p. 50).

Se eligió como fecha el primer fin de semana de noviembre, coincidente con las fiestas del Día de Muertos, una decisión que probablemente tenía tintes mercadológicos. Aunque el evento agotó su boletaje (Rosas, 2015, p. 41), la transmisión televisiva por el Canal 4 de Televicentro estuvo disponible para los aficionados que quedaron fuera («I Gran Premio», 1962a). Por otra parte, la cobertura de la prensa se inició apenas los pilotos y los coches arribaron al país, aproximadamente una semana antes de la carrera (Hernández, 1962a, 1962b, 1962c, 1962d).

A pesar de la muerte de Ricardo Rodríguez durante las jornadas de prácticas (Jalife Villalón, 2015, pp. 298-300), la carrera no fue cancelada. El día del evento, se llenaron las gradas del Autódromo, se depositó una ofrenda floral en el lugar del accidente del piloto mexicano y se lo homenajeó, con Adolfo López Mateos encabezando los actos protocolarios («Solemne ceremonia presidida», 1962).

La primera carrera de Fórmula 1 en México fue un éxito comercial para sus organizadores, y la FIA acordó incluirla como prueba oficial a partir de la temporada 1963 (Hernández Padilla, 2015, p. 34). Mientras, la pista, renombrada de forma no oficial como Autódromo Ricardo Rodríguez («I Gran Premio», 1962b), se convirtió en uno de los inmuebles deportivos más importantes del país.

El Gran Premio de México puede pensarse como un antecedente importante para la celebración de otros espectáculos deportivos masivos, tanto en México como en América Latina. La tendencia continuó durante los siguientes años, pues a mediados de 1963 la ciudad de México presentó oficialmente su candidatura para albergar los Juegos Olímpicos de 1968; «curiosamente, en los días en que se corrió el II Gran Premio, el Comité Olímpico Internacional anunció que los XIX Juegos Olímpicos se realizarían en México» (Rosas, 2015, p. 55).

Por ocho años se celebró de forma ininterrumpida el Gran Premio de México. Durante ese tiempo la asistencia varió poco, aun con la llegada a la presidencia de Gustavo Díaz Ordaz, un personaje con menor arraigo popular que su antecesor y que tampoco parecía disfrutar mucho de los deportes. En 1964 se recibió la noticia de la elección de México como anfitrión del Campeonato Mundial de Fútbol de 1970 («Mundial fútbol», 1964), algo que confirmó a la capital del país como un importante centro del deporte 
espectáculo para la década en curso. Por su parte, la prensa declaró que todo ello había sido posible gracias a «la estabilidad político-económica de nuestro país, y aún más la simpatía que inspira al mundo entero» («Mundial fútbol», 1964).

Como otros espectáculos deportivos, el Gran Premio de México estuvo impregnado de diferentes narrativas que dan cuenta de los problemas políticos, económicos y sociales del país, pero también de las estructuras (principalmente ideológicas) que lo hicieron posible. Una de las ideas que se difundían por los medios de comunicación se relacionaba con la (supuesta) eliminación de las barreras sociales y económicas entre los mexicanos. La carrera se ufanaba de reunir «gente de todas las clases sociales y edades» («Sr. Presidente», 1965). Este era también el principal discurso legitimador del evento, repetido desde el comité organizador: «Propósito fundamental de los organizadores del IV Gran Premio de México es presentar un espectáculo para las mayorías» («Propósito fundamental», 1965).

A pesar de ello, cuando nos detenemos a analizar las condiciones de acceso al evento, caemos en la cuenta de una importante contradicción. En primer lugar, cabe destacar que la venta de los boletos se hacía en comercios establecidos, propiedad de patrocinadores asociados al evento, como en el caso de las concesionarias Ford («IV Gran Premio», 1965), lo que ya condicionaba el acceso a determinados patrones de consumo material y cultural. A pesar de la cobertura periodística (limitada tecnológica y materialmente), el precio y la disponibilidad de cierto número de boletos son otros factores que cierran el acceso masivo a este espectáculo deportivo, a pesar de su popularidad.

Así, el autódromo se convierte en un entorno cerrado, prácticamente de forma hermética, y que es igual tanto para los competidores como para los espectadores. El acceso a este espacio se racionaliza al extremo fetichizando el boleto de acceso, un (nuevo) artículo de lujo de la sociedad deportizada, cada vez más común según se van creando más espectáculos deportivos a lo largo del país. Con ello, el tiempo y las actividades de ocio se socializan como un privilegio que contribuye a la desigualdad.

El Feliz Poseedor de Boleto goza no tanto por saberse allí, sino por la piedad que le dedica a los ausentes. Benditos ellos, no tuvieron dinero y no les quedó sino confiar en las bondades de la televisión, que lo reduce todo a las proporciones [y posibilidades] de una cajita. (Monsiváis, 2013, p. 205)

El boleto de acceso a este y otros espectáculos deportivos se va convirtiendo en una mercancía cada vez más codiciada. Con mayor frecuencia, es blanco de falsificaciones, exhibidas y censuradas por la prensa: 
A varios grandes aficionados al automovilismo se les ocurrió una cosa muy original: falsificar los pases para la entrada fácil a los pits... Ayer fueron sorprendidos en el Autódromo y remitidos a la delegación más cercana. («Boletos falsos», 1967)

Mientras, el espectáculo se antojaba (contradictoriamente) cada vez más exclusivo ante la mirada atenta de la socialité mexicana, cuya presencia se delataba en las páginas de sociales de los periódicos del fin de semana como El Universal y Novedades. Fuera de las páginas rosa de la prensa, el movimiento de las masas -las confinadas en las gradas, pero especialmente aquellas excluidas del ocio deportivo - se convierte en una fuerza opositora (no necesariamente crítica). Ante la amenaza que las masas representan (Varela, 2014, p. 180), la comunidad automovilística, que se identifica plenamente con las ideas y los valores que ostentan estos eventos, invoca la presencia de la fuerza pública para mantener «el orden»: «[Lo bueno es] que se haya utilizado la policía militar para mantener el orden [...] en todo lugar de la Deportiva» («II Gran Premio», 1963). Las interacciones entre los vigilantes y los faltos de boleto también llegaron a ser violentas: «Hubo chamacos que para estar más cerca de sus ídolos, saltaron las bardas, ahí con lujo de fuerza los policías los agarraban para llevarlos a la delegación» («Bastantes curiosos», 1963).

La vigilancia del espacio se vuelve cada vez más estricta y llega a generar incidentes con las personas que trataban de entrar sin boleto a la carrera. En ocasiones, el personal de seguridad, que contaba con policías y soldados entre sus filas (Rosas, 2015, p. 85), se veía rebasado:

Jack Brabham ${ }^{8}$ declaró que lo molestaron varios espectadores que se colocaron casi al borde de la pista. Es que fue casi imposible dominar la tremenda muchedumbre que se desparramó en la Magdalena Mixhuca. («V Gran Premio», 1966)

En otras ocasiones, los incidentes entre espectadores y los cuerpos de seguridad del autódromo se debían a la falta de familiaridad de los primeros con la efímera lógica de desplazamiento organizada a través del boleto de acceso:

Fue una carrera «blanca» y solo hay que lamentar que los organizadores pongan gente sin criterio al cuidado de las puertas. El público fue víctima de vejaciones sin cuento y los periodistas se vieron obstaculizados para cumplir su misión. (Hernández, 1966, p. 9) ganar dentro de la Fórmula 1 con un coche construido por ella misma (Rosas, 2015, p. 50). 
Finalmente, parece que la exclusión se legitima en segundo momento a través de la caracterización de los infiltrados como elementos peligrosos para las actividades en la pista:

Grupitos de espectadores que ayer, en el VI Gran Premio de México, no guardaron la debida compostura y pusieron en peligro su propia vida y la de los corredores. («Grupitos espectadores», 1967)

Aunque el Gran Premio de México no fue pionero en el deporte espectáculo mexicano, es posible observar en él algunas características con las que podemos definir de manera general los espectáculos deportivos propios de un mundo avanzado en su proceso de globalización. Entre estas características se encuentran: 1) la acción conjunta entre las élites políticas y económicas que 2) conciben (e imprimen) una importante dimensión ideológica al evento, donde se enaltecen valores nacionalistas y 3) valores modernizadores que tienden a la reforma de la vida social en su totalidad en busca de un bienestar material basado en la racionalidad capitalista.

Como espectáculo deportivo, el Gran Premio de México parece haber trasladado a un segundo plano los vínculos afectivos alrededor de la actividad de correr automóviles, para que fueran sustituidos por una nueva lógica de excepcionalidad donde lo más importante era la posesión del pase de acceso al espacio diferenciado. La racionalización del espacio lleva también a una racionalización del comportamiento y la creación de códigos de conducta que, paradójicamente, constriñen la liberación emocional que acompaña a la actividad deportiva.

\section{La nación mexicana vista desde el deporte motor}

Por otro lado, existe también una importante dimensión retórica inserta en el Gran Premio de México. En ella se exhiben ideales nacionalistas y modernizadores que muestran un proyecto nacional con el que se identifica la comunidad automovilística. Los ideales de la modernización y el sentimiento patriótico estuvieron impregnados (no necesariamente de forma consciente) en prácticamente cada rincón de la organización del Gran Premio de México. A partir de estos elementos, se articula una propuesta del deber ser de lo nacional, así como de sus elementos constitutivos.

De esta manera, la construcción de la nación mexicana a partir del espectáculo deportivo involucró no solo los elementos estéticos que formaron parte de la identidad gráfica de cada uno de estos espectáculos. También incluyó elementos ideológicos derivados de las transformaciones políticas y económicas que vivía el país para construir un cuerpo discursivo propio. En este proyecto ideológico, generado a la par del desarrollo 
estabilizador, el Gran Premio de México se convirtió (discursivamente) en un elemento parte de la narrativa del milagro mexicano. En este, como en otros eventos deportivos de la época, se imprimió un orgullo patriótico manifestado en los diferentes actores y elementos directamente relacionados con la carrera.

El escenario de la competencia, el Autódromo de la Magdalena Mixhuca, contenía en sí mismo valores nacionalistas desde su propia concepción. El ingeniero Óscar Fernández Gómez Daza, quien concibió por primera vez la construcción de un autódromo en la capital del país, justificó su proyecto basándose en la popularidad del deporte motor en México, así como las condiciones económicas y políticas que, en su opinión, volvían viable la construcción de un inmueble deportivo con lo último en la tecnología de la época, con lo que se podría colocar al país a la cabeza en la disciplina automovilística (Fernández Gómez-Daza, 1955, pp. 3-4).

Como espectáculo deportivo, el Gran Premio de México se estructuró también como una exposición del orgullo nacionalista mexicano. Desde su primera edición se acomodó la celebración de la carrera para que coincidiera con el Día de Muertos, lo que puede considerarse como parte de un discurso diferenciador, paradójico, dada la homogeneización cultural que supone la adhesión a la modernidad. El evento buscaba participar en un diálogo entre naciones iguales entre sí. Esto se volvió prácticamente una política de Estado, nacida cuando el presidente Adolfo López Mateos encargó a los organizadores de la carrera que fueran «portavoces y embajadores del evento que deberá posicionar internacionalmente a México» (Jalife Villalón, 2015, p. 234).

Por otra parte, la narrativa alrededor del Gran Premio de México contiene también elementos que remiten a las tradiciones y raíces culturales de la sociedad mexicana (identificadas en la gastronomía, vestimenta, costumbres, etcétera). Sin embargo, estos componentes cumplieron una función mayormente estética, como diferenciadora cultural y de acompañamiento. Esto se vuelve más claro a la luz de los acontecimientos. Luego de cada edición del Gran Premio de México, los organizadores ofrecían a los pilotos un festejo privado, nueva ocasión para mostrar el colorido de la cultura mexicana. En 1967, el ganador, Jim Clark, fue protagonista de un episodio singular:

Una fiesta mexicana en el cortijo La Morena de Texcoco enmarcó la noche del domingo la entrega de premios a los pilotos [...] Jim Clark, el vencedor del VI Gran Premio, Graham Hill, Joakim Bonner y Jackie Stewart vistieron el traje campero y se lanzaron al ruedo... ipara lidiar ( $i$ ?) un burro con cuernos! Luego soltaron un pequeño becerro a Clark [...] ante el regocijo de unas 500 personas que asistieron al acto. («Una fiesta», 1967)

Cabe considerar otro elemento simbólico en los hermanos Pedro y Ricardo Rodríguez, máximos exponentes del automovilismo deportivo mexicano de ese momento; 
eran también las figuras que sintetizaban la narrativa nacionalista dentro de la competencia deportiva profesional. Los hermanos, que habían iniciado su carrera deportiva en 1957 (Jalife Villalón, 2015), fueron convertidos en embajadores del país, pero también de su juventud: «ejemplo de la auténtica juventud mexicana — de la juventud deportista, no la decadente que se ahoga en las bajas pasiones del vicio y del crimen» («Ricardo Rodríguez», 1962).

La nación, o por lo menos una versión del proyecto de nación ostentado por los grupos detrás de la celebración del Gran Premio de México, se encarnó en los pilotos mexicanos que participaron en las diferentes ediciones de la carrera. Por ejemplo, la muerte de Ricardo Rodríguez durante los entrenamientos previos generó conmoción en la sociedad urbana, tanto por la presencia de la muerte como, en mayor medida, por la pérdida de una referencia moral. El velorio del joven, hecho en la casa de la familia, estuvo abarrotado por sus seguidores, pues «Ricardo era ya una figura deportiva nacional que se había clavado muy hondo en el corazón de los mexicanos» («Murió Ricardo», 1962).

Con el paso de los años, la carrera se convirtió en un elemento más de la moderna nación lograda por el Estado mexicano, que imprimió a este triunfo ideológico un falso carácter colectivo. Así, el presidente Díaz Ordaz declaró: «Este evento no se debe ni al gobierno de la República ni a mí, sino al entusiasmo del público y a los organizadores» («V Gran Premio», 1966). Cada último fin de semana de octubre, «la imagen de México se proyecta al mundo por medio del deporte automovilístico, gracias a la celebración del Gran Premio de México, que sirve además para acrecentar el conocimiento que se tiene de nuestro país en el extranjero» («VIII Gran Premio»1969).

\section{Conclusiones}

La invención del automóvil es uno de los productos de una revolución industrial y tecnológica que marcó el rumbo del siglo XX. Como parte de esta transformación de escala global, es quizás una de las máquinas que más influyeron en las condiciones materiales de las sociedades. Particularmente en México, como país periférico, su llegada (tardía) aceleró los procesos económicos de industrialización, modificó los flujos de producción y consumo y aceleró la migración del campo a la ciudad, así como otras transformaciones demográficas. Todos estos cambios se vieron acompañados por otros de orden cultural, en los que el automóvil se vuelve protagonista ideológico y material; nace, pues, la sociedad automotriz.

La incursión del automóvil en los tiempos sociales del ocio se cristalizó principalmente a través de las competiciones, donde estas máquinas eran las protagonistas. La posterior y gradual institucionalización de esta actividad alrededor de todo el mundo generó espacios dentro de ella para la expresión (consciente e inconsciente) de valores, 
posturas políticas, modelos y símbolos hegemónicos que permearon en la cultura de la sociedad urbana moderna. A su vez, como un deporte profesional y moderno, el automovilismo deportivo abonó (aunque no exclusivamente) en América Latina un modelo de espectáculo deportivo caracterizado por la coparticipación de entidades públicas y privadas, la racionalización del espacio y las conductas públicas socialmente aceptadas, y la mercantilización en general de la actividad deportiva.

Dentro de la dimensión ideológica del deporte profesional (y del deporte espectáculo) en general, así como del automovilismo deportivo en particular, se encuentran expresiones (conscientes e inconscientes) que se convierten en encomiendas de clases y grupos sociales. Dichas expresiones muestran proyectos de clase que luchan por un espacio en la agenda pública, así como el dominio hegemónico de las autopercepciones sociales. El automovilismo deportivo mexicano de la segunda parte del siglo XX, especialmente a través de la Fórmula 1, exhibe un proyecto nacionalista basado en la modernidad industrial del país y su constitución como un colectivo homogeneizado, que reserva sus componentes tradicionales (entre ellos lo indígena) a papeles más bien estéticos, cuando no invisibilizados.

\section{Referencias bibliográficas}

Avendaño, A. (2019). "A ver quién llega primero...» Automovilismo deportivo y transformaciones de la sociedad mexicana entre 1950 y 1970. (Tesis de maestría). Universidad Nacional Autónoma de México, México.

Avendaño, A. (2021). La carrera panamericana y las transformaciones de la sociedad mexicana en la década de los años cincuenta. En M. J. Garrido Asperó y R. Hernández Franyuti (eds.), El fenómeno deportivo en México 1875-1968: Ensayos sobre su historia social, cultural y política. México: Instituto de Investigaciones Dr. José María Luis Mora.

[Bastantes curiosos]. (1963, 25 de octubre). Novedades, p. 30.

[Boletos falsos]. (1967, 22 de octubre). El Heraldo de México, p. 13.

Cárdenas, E. (2012). La economía mexicana en el dilatado siglo XX, 1929-2010. En S. Kuntz (ed.), Historia mínima de la economía mexicana: 1519-2010 (pp. 232-302). México: El Colegio de México.

de la Peña, S., y Aguirre, T. (2004). De la revolución a la industrialización. En E. Semo (coord.), Historia económica de México. México: UNAM.

Fernández Gómez-Daza, O. (1955). Pista para carreras «Gran Premio» (Tesis de licenciatura). Universidad Nacional Autónoma de México, México.

Garrido Asperó, M. J. (2015). El automovilismo deportivo en México: Sus primeros clubes y competencias (siglo XX). Historia Crítica, (61), pp. 105-123. 
Gracida, E. (2004). El desarrollismo. En E. Semo (coord.), Historia económica de México. México: UNAM.

[Grupitos de espectadores]. (1967, 23 de octubre). Excélsior, p. 3.

Hernández, A. (1962a, 23 de octubre). Vienen los pilotos Ireland y Gregory con sendos Lotus. ESTO.

Hernández, A. (1962b, 24 de octubre). Bruce McLaren y Penske al Gran Premio de México. ESTO.

Hernández, A. (1962c, 24 de octubre). Los Hnos. Rodríguez arribaron a México. ESTO.

Hernández, A. (1962d, 25 de octubre). Moisés Solana piloteará un Cooper en el Gran Premio. ESTO.

Hernández, A. (1966, 24 de octubre). Brabham no pudo dar caza a Surtees. ESTO.

Hernández Padilla, J. A. (2015). Así fue... La Fórmula 1 en México 1962-1970 1986-1992. León: Multilibros.

[I Gran Premio de México]. (1962a, 31 de octubre). Novedades, p. 15.

[I Gran Premio de México]. (1962b, 5 de noviembre). El Universal, p. 1.

[II Gran Premio de México]. (1963, 28 de octubre). El Universal, p. 14.

[IV Gran Premio]. (1965, 20 de octubre). ESTO, p. 3.

[V Gran Premio de México]. (1966, 30 de octubre). El Heraldo de México, p. 1.

[VIII Gran Premio de México]. (1969, 18 de octubre). Novedades, p. 2.

Jalife Villalón, C. (2015). Los hermanos Rodríguez. México: Scudería.

Jáuregui, L. (2004). Los transportes: siglos XVI al XX. En E. Semo (coord.), Historia económica de México. México: UNAM.

Juárez Núñez, H., Lara Rivero, A., y Bueno Castellanos, C. (coords.) (2005). El auto global: Desarrollo, competencia y cooperación en la industria del automóvil. México: BUAP.

Loaeza, S (1993). La sociedad mexicana en el siglo XX. En J. Blanco y J. Woldenberg (eds.), México a finales de siglo: Tomo I (pp. 108-120). México: Fondo de Cultura Económica.

Monsiváis, C. (2013). Entrada libre: Crónicas de la sociedad que se organiza. México: Era. [iEl mundial de fútbol!]. (1964, 8 de octubre). ESTO, p. 3.

[¡Murió Ricardo!]. (1962, 1 de noviembre). El Universal, p. 1.

Plana, M. (2004). Las industrias: Siglos XVI al XX. En E. Semo (coord.), Historia económica de México. México: UNAM.

Pedraza Velásquez, J. M. (2011). La otredad mexicana en Memín Pinguín: Un acercamiento al México posrevolucionario. Recuperado de https://pepines.iib.unam.mx/ ensayo/7.

Pozas Horcasitas, R. (2014). Los límites del presidencialismo en las sociedades complejas. México en los años sesenta. México: Siglo XXI.

[Propósito fundamental]. (1965, 24 de octubre). Novedades, p. 2. 
Ramos Torres, R. (1994). Del aprendiz de brujo a la escalada reflexiva: El problema de la Historia en la Sociología de Norbert Elias. REIS: Revista Española de Investigaciones Sociológicas, (65), 27-53.

[Ricardo Rodríguez]. (1962, 4 de noviembre). Novedades, p. 15.

Rosas, A. (2015). Héroes al volante: la Fórmula 1 en México. México: Planeta.

[Solemne ceremonia presidida]. (1962, 5 de noviembre). ESTO, p. 2.

Scudería Hermanos Rodríguez. (2007). Salón de la fama. Recuperado de http://www.sportcar.com/scuderia/votacion/listado_2007.asp.

[Sr. Presidente]. (1965, 25 de octubre). ESTO, p. 4.

Tello, C. (1979). La política económica en México 1970-1976. México: Siglo XXI.

Tovalín Ahumada, A. (coord.) (2005). Y México se subió al camión: Caminos y camiones de México. Ensayo fotográfico. México: Comisión de Transportes.

Varela Hernández, S. (2014). No queremos goles, queremos frijoles. En F. Carrión y M. J. Rodríguez (eds.), Luchas urbanas alrededor del fútbol (pp.171-194). Quito: 5. a Avenida.

[Una fiesta]. (1967, 24 de octubre). ESTO, 24. 